\title{
25 Research Soure \\ Comparative clinical characteristics among different age group of adult COVID-19 Patients
}

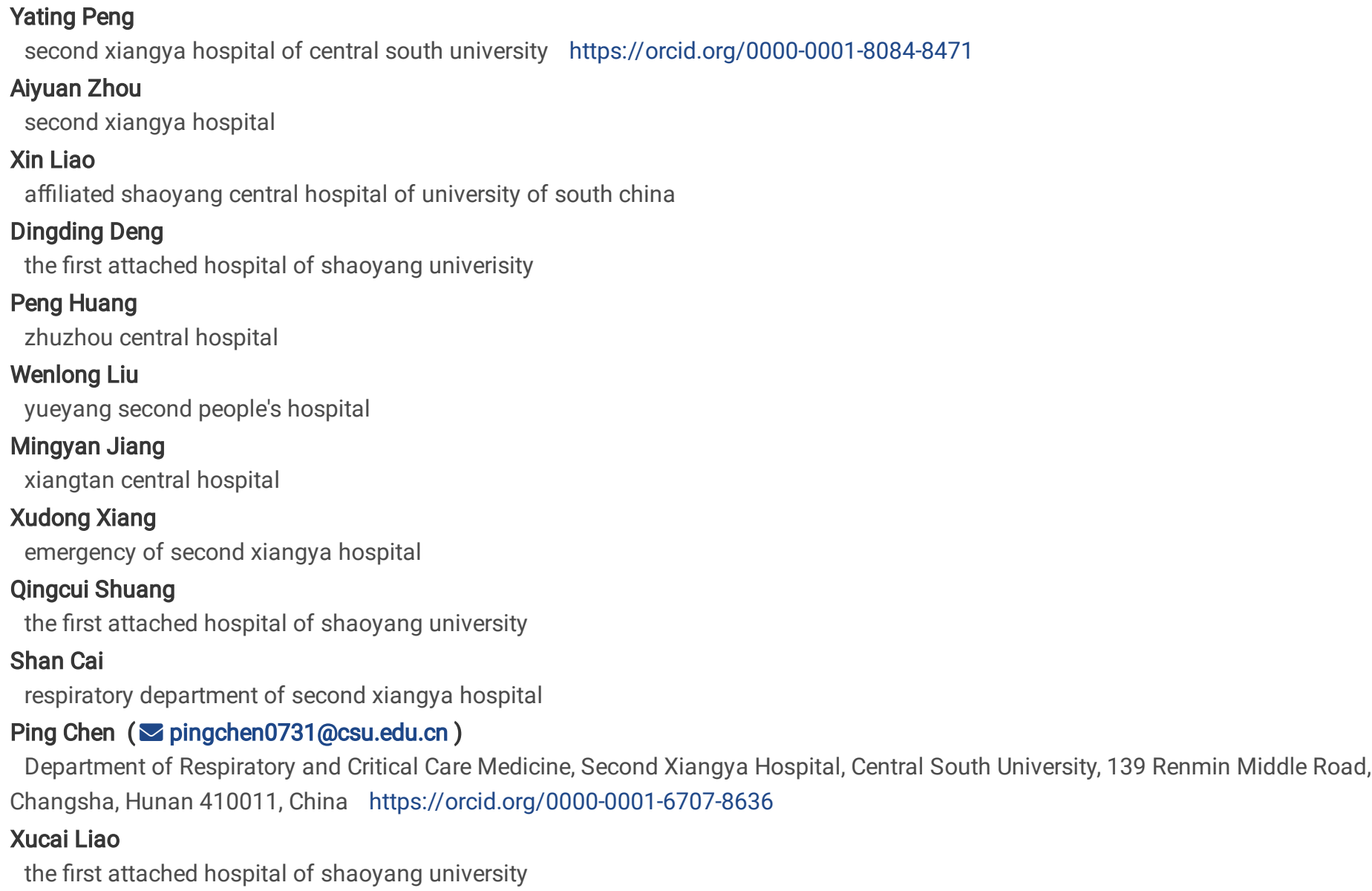

Research

Keywords: COVID-19, severe or critical type, age

Posted Date: April 29th, 2020

DOI: https://doi.org/10.21203/rs.3.rs-23684/v1

License: (c) (7) This work is licensed under a Creative Commons Attribution 4.0 International License. Read Full License 


\section{Abstract}

Introduction: Coronavirus disease (COVID-19) is a global infectious disease with a large burden of illness and high healthcare costs.

Objectives: This study aimed to compare clinical features among adult COVID-19 patients in different age groups.

Methods: Laboratory-confirmed adult COVID-19 infection cases between Dec 31, 2019 to March 8, 2020 obtained from Neighboring Cities. Patients were divided into five age groups: age $₫ 30,30-40,40-50,50-65, \geq 65$ y (elderly). Age, sex, history of chronic disease and epidemiology, symptoms, laboratory tests, and outcomes were compared among different age groups. Binary logistic regression analysis was conducted to evaluate associated factors for severe or critical type.

Results: We studied 299 cases. Median (IQR) age was $44(34,54)$ and $158(53 \%)$ were male. Percent of bilateral involvement on chest radiographs was increased significantly with older age $(p=0.005)$. $53.3 \%$ of $30-40$ years, $50 \%$ of $40-50$ years, $36.6 \%$ of $\varangle 30$ years and $36.2 \%$ of 50-60 years were imported case, none of the elderly were imported case. Among all the observed symptoms, only symptom of dyspnea was significantly different between the elderly group and other groups $(p<0.001)$. Proportion of severe or critical type was $2.4 \%, 5.3 \%, 9.5 \%$, $14.5 \%$, and $35 \%$ in patients with age $\varangle 30,30-40,40-50,50-65, \geq 65(p<0.001)$, respectively. At this point, ICU admission rate, ARDS and shock rate and on medical treatment rate was increased especially in patients $\geq 65$ years. 285 patients (95.3\%) were cured and discharged, 12 patients $(4.0 \%)$ were still on medical treatment in hospital. There were $2(0.67 \%)$ deaths; these occurred among persons $\geq 65$ y $(p<$ 0.001). Old age, high HR on admission, high respiratory rate on admission, and history of chronic heart disease were independently associated with severe or critical .

Conclusions: Proportion of severe or critical type increased with old age groups. Adults with old age and high HR, R rate in admission and history of chronic heart disease were associated with severe or critical type in COVID-19.

\section{Background}

The current outbreak of coronavirus disease (COVID-19) pandemic that was first reported from Wuhan, China, on 31 December 2019 has developed into a world-wide public health emergency. The global number of confirmed cases of COVID-19 has surpassed 600000 as of 28.March.2020 [1]. In China, epidemiological evidence suggested that most of these patients had travelled to Wuhan city (Hubei province) before the onset of illness [2]. Around China's spring festival in early February, rapidly increasing new cases were identified in Hubei and neighboring provinces and cities. Until 8 March 2020, cumulative reported confirmed cases was 80735 in China, and cumulative deaths were 3119 [3], while cumulative reported confirmed cases was 1018 in Hunan province.

Severity assessment is key in determining patient disposition, appropriate therapies, and resource allocation in epidemic events. For example, in China's experience, the Fang Chang Hospital in Wuhan city is organized to treat large numbers of patients with mild and moderate COVID-19, whereas other facilities with negative-pressure isolation wards and intensive care units (ICU) serve patients with severe and critical COVID-19. Incidence and mortality of community-acquired pneumonia is much greater in the elderly ( $>65 \mathrm{y})$ than in younger populations [4]. Old age itself is an independent risk or prognostic factor for pneumonia [5]. More frequent comorbid illness, less specific presenting clinical signs, and lowered effectiveness of therapy in older subjects also add risk $[4,6]$.

Patients of different ages may have distinct physiological characteristics, susceptibility, clinical presentations, and response to medical treatment [7-9]. Therefore, age- specific risk factors for disease severity may be useful for clinical management. To the best of our knowledge, there has been no comprehensive study regarding exploration of the associated factor of severe or critical type on COVID-19 cases. We report here findings describing the influence of age on clinical and laboratory features from a cross-sectional study of patients treated for COVID-19 in five University or municipal hospitals in Hubei and Hunan provinces.

\section{Materials And Methods}

\section{Study population, data collection, and study design}

A hospital-based COVID-19 disease surveillance program was established and maintained from December 2019 to March 2020. This included 6 participating hospitals (Affiliated Shaoyang Central Hospital of University of South China, The first Attached Hospital of Shaoyang University, Zhuzhou Central Hospital, Designated Hospital of Junshan District, Xiangtan Central Hospital) from Hunan Province and Puyang District People's Hospital from Hubei Province. We included patients $\geq 18 \mathrm{y}$ who were positive for coronavirus nucleic acid detection in throat swab at admission. None of the patients were positive for Influenza A or B virus nucleic acid detection in throat swab. 
This is a cross-sectional study based on review of medical records. Clinical variables including patient demographic data, previous chronic illnesses, epidemiological contact history data, clinical management, and outcome were collected on a standardized case report form at each hospital.

\section{Definitions}

Patient variables were evaluated for all cases: Demographic characteristics (e.g., age, gender, BMI); chronic disease history involving the central nervous system, cardiovascular system, lungs, liver, kidneys, endocrine system, autoimmune conditions; initial vital signs (e.g. temperature and respiratory rate), laboratory test results (White blood cell [WBC], lymphocyte [Lym], neutrophil [Neu], platelets[Pt], hemoglobin [Hb], Prothrombin time [PT time], Activated partial thromboplastin time [APTT], D-Dimer [DD], albumin, alanine aminotransferase [ALT], aspartate aminotransferase [AST], Creatine kinase [CK], Creatine kinase isoenzyme [CK-MB], Total bilirubin [TBIL], $\mathrm{K}+, \mathrm{Na}+$, Urea nitrogen [BUN], creatinine [Cre], blood cell sedimentation rate [ESR], Lactate dehydrogenase [LDH], Myoglobin [Mb], Random blood glucose [Glu], c-reactive protein [CRP], Procalcitonin [PCT], chest radiography); and medications. Data on initial vital signs, laboratory tests and chest radiography findings were from the first readings taken in the hospital.

For epidemiological history, patients with Wuhan travel history were defined as imported cases; patients without Wuhan travel history, but with contact with imported cases or confirmed cases, were defined as secondary cases, the remainder without definite epidemiologic linkage were classified as "other."

COVID-19 severity was classified into four categories. Mild: Mild clinical symptoms (cough, fatigue, dyspnea) without imaging findings of pneumonia. Moderate: Fever (admission temperature $\geq 39^{\circ} \mathrm{C}$ and respiratory symptoms with imaging findings of pneumonia. Severe: Having any of the following criteria: 1) Respiratory distress with respiratory frequency $\geq 30$ breaths/min; 2) Resting oxygen saturation $\left(\mathrm{SpO}_{2}\right) \leq 93 \%$ on room air; 3) $\mathrm{PaO}_{2} / \mathrm{FiO}_{2} \leq 300 \mathrm{mmHg}(1 \mathrm{mmHg}=0.133 \mathrm{kPa})$. Critical: Having any of the flowing criteria: 1$)$ Respiratory failure in need of mechanical ventilation; 2) Shock; 3) With other significant organ dysfunction [10].

Outcome was classified as cured and discharged, undergoing treatment in hospital, and deceased in-hospital. Patients were discharged from hospital upon meeting the following criteria: 1). Body temperature returned to normal for more than 3 days; 2) Respiratory symptoms improved significantly; 3 ) Improvement in pulmonary radiographic findings; 4) Two negative throat swabs for coronavirus nucleic acid taken 24 hours apart [10].

\section{Statistical analysis}

Categorical variables were expressed as counts and percentages. Continuous variables were expressed as mean \pm standard deviation or median with $25^{\text {th }}-75^{\text {th }}$ interquartile range (IQR) according to normally or non-normally distributed. Differences in frequencies were compared using chi-square test or Fisher's exact test. In the comparison of continuous numerical variables in independent groups, the Mann-Whitney $\mathrm{U}$ test was used in the case of two groups, whereas nonparametric tests with multiple independent samples corrected by Bonferroni were used three or more groups. Factors with significant $(p<0.05)$ unadjusted associations with disease severity and suspected important variables were included in subsequent multivariable logistic regression analysis (Forward method) to determine risk factors associated with disease severity, yielding adjusted odds ratios (ORs) and $95 \%$ confidence intervals. Goodness of-fit was tested using the Hosmer-Lemeshow test [reference]. All statistical tests were two-tailed, and $p<0.05$ was considered to be statistically significant. All analyses were performed using IBM SPSS Statistics version 18.0 for Windows (IBM Corp, Armonk, NY, USA).

\section{Results}

\section{Demographic and clinical characteristics}

Ages ranged from 18 to $88 \mathrm{y}$ with a median of $44 \mathrm{y}$ (IQR 34-54), and half of participants were male (Supplement Figure 1). Half of patients were in the 30-50-y age groups (Supplement Figure 1). Height was decreased significantly decreased with older age $(p=0.001)$, but BMI was not significantly different among groups.

One-third of participants had one or more chronic disease. The total number of chronic diseases differed significantly $(p<0.001)$ between age groups, with elderly have more chronic disease. Older age groups had more patients with at least one or more chronic disease $(5 \%, 16 \%$, $34 \%, 52 \%$ and $75 \%$ in $\varangle 30,30-40,40-50,50-65$ and $\geq 65$ age groups, respectively; $p<0.001)$. Older age groups had higher prevalences of DM history $(p<0.001)$ and hypertension history $(p<0.001)$, chronic heart disease history $(p=0.013)$, and chronic pulmonary disease history $(p=0.011)$. There was no significant difference in prevalences of history of cancer, chronic nervous system disease, chronic liver and kidney disease, endocrine and immune disease among the age groups. Prevalence of bilateral radiographic pneumonia findings was high in all five 
age groups (overall, $79 \%$ ) and was increasedsignificantly with older age $(61 \%, 73 \%, 82 \%, 86 \%$ and $90 \%$ in $\varangle 30,30-40,40-50,50-65$ and $\geq 65$ age groups, respectively; $p=0.005)$. 71(23.75\%) patients could not provide a definite contact or exposure history with confirmed or suspected COVID-19 infection. $53.33 \%$ of $30-40$ years and $50 \%$ of $40-50$ years were imported cases, and none of the elderly were imported cases. Percentage of secondary cases were $55 \%$ among elderly, $42.03 \%$ in $50-65$ years and $43.9 \%$ in $\varangle 30$ years. Temperature, heart rate, respiratory rate on admission were slightly increased in older age groups (Table 1).

\section{Symptoms}

The most common symptoms were cough in 216 (72\%) patients, fever in $202(68 \%)$, expectoration in 139 (46\%), fatigue in $117(39 \%)$, dry cough in $77(26 \%)$ and were of comparable prevalence in all age groups. Only dyspnea was significantly different between elderly group and other groups $(p=0.001)$ (Supplement Figure 2a).

\section{Comparison of laboratory testing profile}

Hemoglobin ( $\mathrm{Hb}$ ) and albumen levels (Supplement Table 1) fell with increasing age: $\mathrm{Hb} 146,140,141,126$ and 122g/l in $\varangle 30,30-40,40-50,50-$ 65 and $\geq 65$ y age groups, respectively; $p=0.000$; and albumin $42.25,45.36,45.36,42.6,40.9$ and $36.92 \mathrm{~g} / \mathrm{l}$ in囚30, 30-40, 40-50, 50-65 and $\geq 65$ age groups, respectively; $p=0.000$ ).

Patients age $\geq 65$ had significantly higher neutrophil percentage compared to age $₫ 30(69.25 \%$ vs. $57.8 \%, p=0.002)$. Age $\geq 65$ had significant lower lymphocyte percentage compared to age $₫ 30$ and age $30-40$ (19.25 vs. $32.75, p=0.003 ; 19.25$ vs. $29.1, p=0.015)$. Age $₫ 30$ had significantly higher levels of lymphocyte counts compared to age $30-40,40-50,50-65, \geq 65$ (1.6 vs. $1.2, p=0.009 ; 1.6$ vs. $1.15, p=0.002 ; 1.6$ vs. $1.29, p=0.021 ; 1.6$ vs. $1.06, p<0.001)$. The level of ESR and PCT of age $\geq 65$ were the highest among all groups; these values were higher than level of ESR and PCT of age $₫ 30,30-40,40-50$ and 50-65 with statistically significant differences $(p<0.001)$. Age $\geq 65$ had significantly higher levels of CRP compared to age 30-40 (13.9 vs. 0.5, $p=0.002)$. The increased inflammation as reflected by increased ESR and PCT and decreased immune function as reflected by decreased lymphocyte counts suggesting that there may be more inflammatory factor storm and immune dysfunction with increasing age groups.

Measurements such as BUN (5.12 vs. $3.82, p=0.008 ; 5.12$ vs. $3.7, p=0.001 ; 5.12$ vs. $3.7, p=0.018)$ and $\mathrm{Mb}(86.25$ vs. $37.35, p=0.006$; 86.25 vs. $35, p=0.007 ; 86.25$ vs. $33.5, p=0.004$ ) of age $\geq 65$ were significantly higher than those of age $₫ 30,30-40$ and $40-50$.

In addition, levels of DD of age $\geq 65$ was significantly higher than those of age $\rrbracket 30,30-40$ and $40-50$ and $50-65$ ( 0.66 vs. $0.29, p<0.001 ; 0.66$ vs. $0.28, p<0.001 ; 0.66$ vs. $0.31, p<0.001 ; 0.66$ vs. $0.35, p<0.001)$. Levels of platelets of age $\geq 65$ and $50-65$ was significantly lower than those of age $\varangle 30$ ( 175.5 vs. $245, p=0.037 ; 184$ vs. $245, p=0.019$ ) (Supplement Table 1 and Figure 1 ). These changes suggesting potentially heart, kidney and coagulation function damage in the oldest age group. The levels of WBC, N, PT time, APTT, AST, CK, CKMB, TBIL, K, Na, Cr, $\mathrm{LDH}$, and Glu of the groups were not statistically different among different age groups.

\section{Medical treatments and outcomes of COVID-19 among the age groups}

$80 \%$ received Interferon alpha inhalation (239/299), $76 \%$ of the patients received Lopinavir/ritonavir (228/299), $57 \%$ received Arbidol (169/299), and 65.22\% received Lianhua Qingwen Capsule treatment (chinese medicine). Combination use of Arbidol, Lopinavir/ritonavir or Interferon alpha inhalation was also common. Empirical antibiotic treatment was used when bacterial infection was suspected, which may reference for elevated Neu, PCT value and sputum. $42 \%$ were received antibiotic (126/299). Percentage of corticosteroid $(17 / 57,43 \%)$ and gamma globulin $(19 / 56,48 \%)$ treatment was highest in age $\geq 65$.

In particular, mild type accounted for $10.7 \%$ (32/299), moderate type accounted for $77.26 \%$ (75/299), severe type accounted for $8.7 \%$ (10/299), critical type accounted for 3.34\% (4/299). Proportion of severe or critical type was $2.44 \%, 5.33 \%, 9.46 \%, 14.49 \%$, and $35 \%$ in patients with age $\varangle 30,30-40,40-50,50-65, \geq 65$, respectively ( $p<0.001$ ). Proportion of mild or moderate type was $97.56 \%, 94.67 \%, 90.54 \%$, $85.51 \%$, and $65 \%$ in patients with age $₫ 3030-40,40-50,50-65, \geq 65$. The medium time from onset treatment to throat swab turn negative was 15.5 days in age $\geq 65,16$ days in age $50-65$, and 11 days in age $₫ 30,10$ days in $30-40,11.5$ days in $40-50$.

At this point, ICU admission rate was $0 \%, 4 \%, 3 \%, 4 \%$ and $18 \%$ in age $₫ 30,30-40,40-50,50-65, \geq 65(p=0.003)$. ARDS rate was $0 \%, 4 \%, 7 \%$, $9 \%$ and $30 \%$ in age $₫ 30,30-40,40-50,50-65, \geq 65(p<0.001)$. Rate of shock was $0 \%, 1 \%, 1 \%, 0 \%$ and $10 \%$ in age $\varangle 30,30-40,40-50,50-65, \geq 65$ $(p=0.006) .285$ patients $(95.32 \%)$ were cured and discharged, 12 patients $(4.01 \%)$ was still on medical treatment in hospital, 2 patients $(0.67 \%)$ died because of respiratory failure $(p<0.001)$. On medical treatment rate was $0 \%, 1.33 \%, 2.7 \%, 2.9 \%$ and $17.5 \%$ in age $\varangle 30,30-40,40$ $50,50-65, \geq 65$. There were no difference in time from symptom onset to initial treatment and hospitalization days among age groups (Supplement Table 1). 
Patients developed to severe or critical type were older than patients with mild or moderate type (57.72 vs. $44.06 p=0.002)$. Comparison were performed to determine factors associated with severe or critical type in overall COVID-19 patients. Patients developed to severe or critical type have higher percentage of cough ( $94.44 \%$ vs. $69.2 \%, p=0.004)$, fever $(88.89 \%$ vs. $64.64 \%, p=0.002)$, expectoration $(63.89 \%$ vs. $44.11 \%, p=0.026)$, fatigue ( $61.11 \%$ vs. $36.12 \%, p=0.004)$, dyspnea ( $58.33 \%$ vs. $8.37 \%, p<0.001)$, and hemoptysis $(8.33 \%$ vs. $0.76 \%, p=$ 0.014) symptom than patients with mild or moderate type (Supplement Figure $2 b$ ).

In general, patients developed to severe or critical type have higher percentage with at least one chronic disease $(77.78 \%$ vs. $29.28 \%, p<$ $0.001)$, DM ( $30.56 \%$ vs. $9.13 \%, p=0.001)$, chronic heart disease $(22.22 \%$ vs. $2.28 \%, p<0.001)$ and chronic pulmonary disease $(16.67 \%$ vs. $4.18 \%, p=0.009$ ) compared to patients with mild or moderate type. Imported and secondary case accounts for $40.68 \%$ and $38.40 \%$ in patients with mild or moderate type, however, this proportion was $27.78 \%$ and $27.78 \%$ in severe or critical type. Noticing that $44.44 \%$ of severe or critical type was infected with unknown origin of infected.

Besides, relatively high percent of drugs was prescribed in severe or critical type than mild or moderate type. Obviously, severe or critical type have longer hospitalization days ( 20.89 vs. $17.18, p=0.011$ ), higher rate of ICU admission ( $25 \%$ vs. $2.28 \%, p<0.001)$, ARDS (52.78\% vs. $2.66 \%, p<0.001)$, and shock $(13.89 \%$ vs. $0.38 \%, p<0.001)$ than mild or moderate type. Still on medical treatment rate was $19.44 \%$ in severe or critical type and $3.07 \%$ in mild or moderate type. In-hospital death cases were 2 in severe or critical type and none in mild or moderate type. However, there was no significant difference regarding sex, BMI, temperature in admission, bilateral involvement on chest radiographs, other onset symptoms and time from symptom onset to initial treatment and time from onset treatment to throat swab turn negative between severe or critical type and mild or moderate type (Table 3).

\section{Factors for severe or critical type in COVID-19 patients}

In a binary logistic regression analysis, eight factors (age, HR in admission, R in admission, history of DM, HBP, chronic heart disease, chronic lung disease, epidemiological history) were included as covariates. We found old age (OR 1.055, 95\% Cl 1.016-1.095, $p=0.006$ ), HR in admission (OR 1.085, 95\% Cl 1.03-1.144, $p=0.002$ ), $\mathrm{R}$ in admission (OR 1.635, 95\% Cl 1.093-2.431, $p=0.017$ ), and history of chronic heart disease (OR 56.038, 95\% Cl 2.764-1136.053, $p=0.009$ ) showed independent associations with severe or critical type (Table 4).

\section{Discussion}

To the best of our knowledge, our study is the first to show that old age, heart rate in admission, respiratory rate in admission and history of chronic heart disease were associated with severe or critical type in COVID-19 in district outside Wuhan.

In this study, the fatality of COVID-19 infection was $0.67 \%$. The outcome and clinical characteristics of patients outside of Wuhan differed from those initially reported in patients in Wuhan [11,12]. Meanwhile, in our study, severe or critical type accounted for 12.04\% (36/299), and most of the patients were moderate $87.96 \%$ (263/299). The trends are relatively consistent across the major reporting states in China [12]. Until March23, 2020, Hunan Province had no confirmed cases or no new confirmed cases for 14 consecutive days and was listed as lowrisk urban areas in China. This reflects effective treatment and public health response.

Aging is associated with a progressively weakened immune system, decreased lung performance, and a bad outcome in communityacquired pneumonia (CAP). Although we can't assess the susceptibility of COVID-19 and old age, but we found old age was significantly associated with severe or critical type. In recent published studies, the patients in the progression group were significantly older than those in the disease improvement/stabilization group [13]. What's more, for critically ill adult patients infected with COVID-19 who were admitted to the intensive care unit (ICU) in Wuhan, researchers found older patients (>65 years) with comorbidities and acute respiratory distress syndroms (ARDS) are at increased risk of death [14]. These results indicate that age-related risk factors for a bad outcome should be considered when developing effective medical treatment strategies for older patients.

The patients with severe or critical type also more frequently had cough, fever, expectoration, fatigue, dyspnea, and hemoptysis symptoms. Physicians need to be alert to these symptoms. COVID-19 infection in elderly seems to be a little different with elderly CAP, as the elderly with bacterial pneumonia may lack the typical acute respiratory symptoms, but with gastrointestinal symptoms [6,15]. In our study, for elderly, dyspnea symptoms were more common in elderly and respiratory symptom like cough, dry cough, expectoration, fatigue were common symptoms in elderly. Despite this, altered mental status, a sudden decline in functional capacity, and worsening of underlying diseases is still important for differentiate severe cases in COVID-19. 
Comorbid condition is another well-recognized risk factor for pneumonia mortality [16]. In our study, patients who developed severe or critical type were more likely to have underlying medical comorbidities ( $77.78 \%$ vs. $29.28 \%$ ), including DM (30.56\% vs. $9.13 \%)$, HBP (44.44\% vs. $14.07 \%)$, history of chronic heart disease $(22.22 \%$ vs. $2.28 \%$ ) and history of chronic pulmonary disease ( $16.67 \%$ vs. $4.18 \%)$, compared to mild or moderate type. In the logistic analysis, history of chronic heart disease were independently associated with severe or critical type in the regression analysis. There were also an increase trend in medical comorbidities (DM, HBP, chronic heart and pulmonary disease) with advancing age groups. We also noticed a two fold increase of $\mathrm{Mb}$ levels in elderly group compared to other age groups. It is very likely that coexisting chronic diseases work in a synergic manner, affecting patients' general health condition and resilience. As demonstrated by other studies that myocardial injury is significantly associated with fatal outcome of COVID-19 [17,18].

Physicians and patients probably need to be alert to high or increased heart rate, respiratory rate with COVID-19. As HR and R were all easily available data before patients visit physicians, clear awareness of these factors and understanding of their predictive value would help to predict the disease severity, which may further customise the medical care being provided when needed. Initial vital signs and the results of basic laboratory tests and chest radiography are critical information that is required for clinicians to rapidly understand patients' health condition, in particular the severity of acute illness. Although there were significant increase in levels of N\%, DD, ALT, BUN, Mb, ESR, CRP and $\mathrm{PCT}$ and significant decrease in levels of $\mathrm{L} \%, \mathrm{~L}, \mathrm{Pt}, \mathrm{Hb}$ and albumin in old age group, the majority of these values were still in the normal limit of reference, suggesting subtle and sub-clinical changes of many blood lab tests occur with severe or critical type of infection. The association between old age and severe or critical type COVID-19 might be due to reduced immune function, as reflected by increased neutrophil and decreased lymphoncyte percentages, and poor nutrition, reflected by decreased $\mathrm{Hb}$, albumin, inflammatory factor storm, reflected by increased ESR, CRP and PCT; impaired coagulation function, liver, kidney, heart function, reflected by increased DD, ALT, BUN, $\mathrm{Mb}$ and decreased Pt. Recent study also demonstrated decreased albumin is a risk factor for the progression of COVID-19 pneumonia [13]. And anticoagulant therapy appears to be associated with better prognosis in severe COVID-19 patients meeting sepsis-induced coagulopathy (SIC) or with markedly elevated D-dimer [19]. The author thinks immune regulation, nutrition improvment, Inflammatory response control may be helpful for treatment.

As novel coronavirus were newly spread virus, there are many clinical uncertainty. Choosing an effective anti-virus and antibiotic as well as timely treatment during the management of COVID-19 may may improve outcomes, including direct medical, social, and economic. Randomized controlled double-blind clinical trials may provide treatment insights for COVID-19.

The limitation of our study was that limited cases. National wide study with different races will be more more representative and convincing. Second, although we checked vital signs, epidemiological history, chronic disease histories, we only find old age, heart rate in admission, respiratory rate in admission and history of chronic heart disease were risks for more severe disease. However, beyond these factors listed above, there are several other factors, like viral exposure virulence, such as viral subtype and virulence, viral load, and time from symptom onset to treatment that may influence disease severity.

\section{Conclusion}

Age, heart rate in admission, respiratory rate in admission and history of chronic heart disease are are associated with progression to severe or critical type of COVID-19 and thus provide prognostic information and assist clinicians in alerting high-risk patients.

\section{Abbreviations}

White blood cell:WBC, lymphocyte:Lym, neutrophil:Neu, platelets:Pt, hemoglobin:Hb, Prothrombin time:PT time, Activated partial thromboplastin time:APTT, D-Dimer:DD, albumin, alanine aminotransferase [ALT, aspartate aminotransferase:AST, Creatine kinase:CK, Creatine kinase isoenzyme:CK-MB, Total bilirubin:TBIL, Urea nitrogen:BUN, creatinine:Cre, blood cell sedimentation rate:ESR, Lactate dehydrogenase:LDH, Myoglobin:Mb, Random blood glucose:Glu, c-reactive protein:CRP, Procalcitonin:PCT, oxygen saturation: SpO2, community-acquired pneumonia: CAP, intensive care unit: ICU, acute respiratory distress syndroms: ARDS, sepsis-induced coagulopathy: SIC.

\section{Declarations}

\section{Ethics approval and consent to participate}

Ethical approval and waiver of informed consent was obtained. This research was approved by the local Ethics Committee (2020002).

\section{Availability of data and materials}


The datasets used and/or analysed during the current study are available from the corresponding author on reasonable request.

Competing interestsThe authors have no conflicts of interest to declare. FundingThe authors declared that this study has received no financial support. Authors' contributions

Yating Peng generated the hypothesis, directed the implementation. Yating Peng and Aiyuan Zhou contribute to statistical analyses and wrote the manuscript. Xin Liao, Dingding Deng, Peng Huang, Wenlong Liu, Mingyan Jiang, Xudong Xiang, Qingcui Shuang contribute to data collection. Shan Cai contribute to statistical analyses. Ping Chen, Xucai Liao supervised the field activities and and edited the manuscript. All authors read and approved the final manuscript.

AcknowledgementsThe authors wish to thank the patients described in this study and medical staff caring for them. We also wish to acknowledge the educational and mentorship contributions of the Methods in Epidemiologic, Clinical, and Operations Research (MECOR) program of the American Thoracic Society, with support from the Song Qingling Foundation.

\section{References}

[1] https://www.who.int/emergencies/diseases/novel-coronavirus-2019

[2] Lu H, Stratton C W, Tang Y W. Outbreak of Pneumonia of Unknown Etiology in Wuhan China: the Mystery and the Miracle[J]. Journal of Medical Virology.

[3] National Health Commission of the People's Republic China. The latest situation of new coronavirus pneumonia. http://www.nhc.gov.cn/xcs/yqfkdt/202003/f2c83db9f73d4be5be0dc96af731813c.shtml

[4] Brito V, Niederman M S. How can we improve the management and outcome of pneumonia in the elderly?[J]. 2008.

[5] Lee J Y, Yoo C G, Kim H J, et al. Disease burden of pneumonia in Korean adults aged over 50 years stratified by age and underlying diseases[J]. The Korean journal of internal medicine, 2014, 29(6): 764.

[6] Simonetti A F, Viasus D, Garcia-Vidal C, et al. Management of community-acquired pneumonia in older adults[J]. Therapeutic advances in infectious disease, 2014, 2(1): 3-16

[7] Wang L, Green F H Y, Smiley-Jewell S M, et al. Susceptibility of the aging lung to environmental injury[C]//Seminars in respiratory and critical care medicine. (C) Thieme Medical Publishers, 2010, 31(05): 539-553.

[8] Miller E J, Linge H M. Age-related changes in immunological and physiological responses following pulmonary challenge[J]. International journal of molecular sciences, 2017, 18(6): 1294.

[9] Welte T. Risk factors and severity scores in hospitalized patients with community-acquired pneumonia: prediction of severity and mortality[J]. European journal of clinical microbiology \& infectious diseases, 2012, 31(1): 33-47.

[10]http://www.nhc.gov.cn/xcs/zhengcwj/202003/46c9294a7dfe4cef80dc7f5912eb1989/files/ce3e6945832a438eaae415350a8ce964.pdf

[11] Huang C, Wang Y, Li X, et al. Clinical features of patients infected with 2019 novel coronavirus in Wuhan, China[J]. The Lancet, 2020, 395(10223): 497-506.

[12] Tian S, Hu N, Lou J, et al. Characteristics of COVID-19 infection in Beijing[J]. Journal of Infection, 2020

[13] Liu W, Tao Z W, Lei W, et al. Analysis of factors associated with disease outcomes in hospitalized patients with 2019 novel coronavirus disease[J]. Chinese Medical Journal, 2020.

[14] Yang X, Yu Y, Xu J, et al. Clinical course and outcomes of critically ill patients with SARS-CoV-2 pneumonia in Wuhan, China: a singlecentered, retrospective, observational study[J]. The Lancet Respiratory Medicine, 2020.

[15] Waterer G W, Kessler L A, Wunderink R G. Delayed administration of antibiotics and atypical presentation in community-acquired pneumonia[J]. Chest, 2006, 130(1): 11-15

[16] Marrie T J, Carriere K C, Jin Y, et al. Factors associated with death among adults< 55 years of age hospitalized for community-acquired pneumonia[J]. Clinical infectious diseases, 2003, 36(4): 413-421

Page $7 / 8$ 
[17] Bonow RO, Fonarow GC, O'Gara PT, et al. Association of Coronavirus Disease 2019 (COVID-19) With Myocardial Injury and Mortality[J]. JAMA Cardiol, 2020 Mar 27

[18] Guo T, Fan Y, Chen M, et al. Cardiovascular Implications of Fatal Outcomes of Patients With Coronavirus Disease 2019 (COVID-19)[J]. JAMA Cardiol, 2020 Mar 27

[19] Tang N, Bai H, Chen X, et al. Anticoagulant treatment is associated with decreased mortality in severe coronavirus disease 2019 patients with coagulopathy[J]. J Thromb Haemost, 2020 Mar 27

\section{Supplementary Files}

This is a list of supplementary files associated with this preprint. Click to download.

- Supplement.docx

- Supplement.docx 